\title{
Evanescent-wave Sensing with Multiple Sampling Channels along a Single Optical Fiber
}

\author{
Jianjun Ma, ${ }^{* 1}$ Wojtek J. Bock, ${ }^{1}$ \\ ${ }^{1}$ Centre de recherche en photonique, Département d'informatique et d'ingénierie, Université du Québec en \\ Outaouais, P. O. Box 1250, Stn. Hull, Gatineau, Québec J8X 3X7, Canada.
}

Received September 25, 2013; accepted December 07, 2013; published December 31, 2013

\begin{abstract}
We demonstrate that the evanescent-wave sensing platform with one sampling channel may be expanded to at least a 12-channel system. These channels are implemented as a series of 2-mm long exposed segments of the fiber core sidewall along a single optical fiber. Using the cladded fiber segments as channel separations, we achieve a condition of zero inter-channel crosstalk, which is fundamental to the system operation, for both 4- and 12-channel systems. Theoretical interpretation of the mechanism of zero inter-channel crosstalk is examined in detail.
\end{abstract}

Fiber-optic evanescent-wave (EW) sensing platforms are the powerful tools for assays of chemical and biological samples on the surface layer of the fiber-core sidewall [1]. So far, most fiber-optic EW sensing platforms have had a single sampling channel [1-5]. Fewer reports have shed light on the construction of a multiple-channel system along a single fiber. While in principle a true distributed system can be constructed by measuring the optical pulse delay to pinpoint the interaction region [2], an expensive OTDR is needed to interrogate the weak return signal and to achieve a sufficient spatial resolution. In this paper, we propose an alternative fiber-optic EW sensing platform equipped with a number of sampling channels for multiple sample assays.

Obviously, the multiple channels distributed along a single fiber imply that the captured EW signal from the excited channel may need to pass one or more other channels before arriving at the detector. Thus, the fundamental mechanism required for the operation of the proposed platform is its ability to maintain zero inter-channel crosstalk. Such crosstalk may occur when a sample is added to or removed from these channels. We demonstrate that such a zero inter-channel crosstalk operation could be achieved for the proposed platform.

As shown in Fig. 1, the platform includes a multimode sensing fiber (s-fiber) with multiple built-in sampling channels from $\mathrm{CH} 1$ to $\mathrm{CHn}$. Each channel is a bare core segment approximately 2-mm-long, and the channels are separated by clad segments of arbitrary lengths. Requiring only a few microliters of sample apiece, these channels are

${ }^{*}$ E-mail: ma.jianjun@uqo.ca scanned one at a time by another fiber (the e-fiber), positioned perpendicularly to the s-fiber for excitation light delivery. An alternative approach is to assign a dedicated e-fiber to each channel and to excite one channel at a time via optical switching (the case shown in Fig. 1).

Again, achieving a state of zero inter-channel crosstalk is fundamental to the successful operation of this platform. This is verified when an EW signal at the detector sent from the sample at any channel ( $\mathrm{CH} 2$ in Fig. 1, for instance) is unaffected by the events occurring at any other channel $(\mathrm{CH} 0, \ldots \mathrm{CHj}, \ldots \mathrm{CHn}, \mathrm{j} \neq 2)$. Such events can be simulated by placing test droplets across the channels in a random fashion. We used distilled water for the test droplets since its refractive index (RI) of $n_{l q}=1.33$ is typical of liquid samples in general.

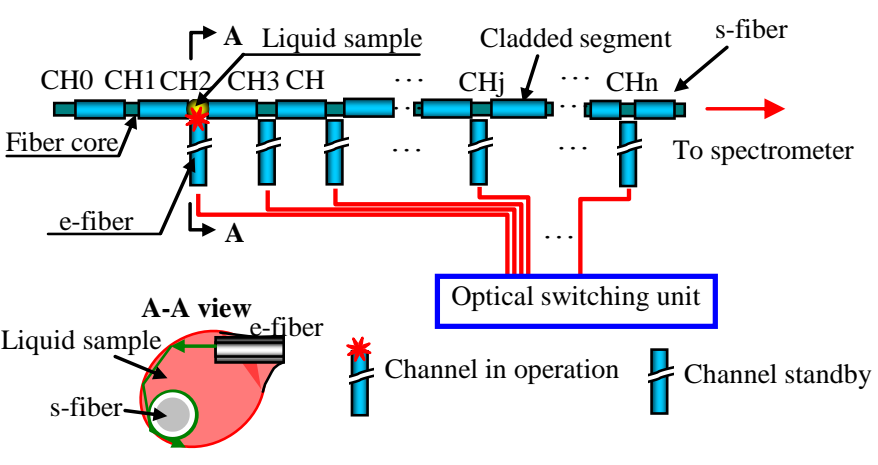

Fig. 1. Principle of operation of the proposed platform. A-A view: the optimum alignment of the two fibers and the sample.

Following Fig. 1, we constructed a 4-channel platform from a two-meter length of BFL37-400 (Thorlabs Inc.) multimode fiber. The channel separation is approximately $1 \mathrm{~cm}$. The fiber core, cladding and jacket have diameters of $400 \mu \mathrm{m}, 430 \mu \mathrm{m}$ and $730 \mu \mathrm{m}$, while the RIs of the core and cladding are $n_{\mathrm{co}}=1.46$ and $n_{\mathrm{cl}}=1.41$, respectively. A rhodamine 6G (R6G) water solution with its spectrum centered at $550 \mathrm{~nm}$ was chosen to represent the fluorescence-capable sample, and was dispensed into $\mathrm{CH} 2$. Another BFL37-400 fiber was used as an e-fiber to deliver the $532 \mathrm{~nm}$ laser beam to the sample. The optimum 
alignment is determined by shaping the R6G drop (see A-A view in Fig. 1) until the maximum fluorescent power is observed at the detector [3], which is a USB2000 spectrometer from Ocean Optics. The test droplets were dispensed into $\mathrm{CH} 0, \mathrm{CH} 1$ and/or $\mathrm{CH} 3$ in a random fashion to verify if there was zero crosstalk.

The inset in Fig. 2, comprising from top to bottom a group of legends with the fibers, illustrates the sequence of adding a test droplet (numbers 1 to 4 ) and removing it (numbers 5 to 7 ) while continuously monitoring $\mathrm{CH} 2$. The nearly perfect overlap of the seven spectra from $\mathrm{CH} 2$ in the presence of these events clearly indicates the absence of inter-channel crosstalk. The minor variations among the spectra are mainly due to fluctuations of the laser excitation power. We further expanded this platform to a 12-channel system and captured the fluorescent spectrum from $\mathrm{CH} 5$. By repeating the same experiment done on $\mathrm{CH} 2$ and shown in Fig. 2, we again proved zero inter-channel crosstalk.

The elimination of the inter-channel crosstalk can be explained by the guided mode capacity of the fiber $N_{\mathrm{g}}$, which is determined by [6]:

$$
N_{g}=\pi^{2} d^{2}\left(n_{c o}^{2}-n_{c l}^{2}\right) / 2 \lambda^{2},
$$

where $d$ and $\lambda$ are the fiber core diameter and the wavelength, respectively.

Using $N_{g, a r}, N_{g, l q}$ and $N_{g, c l}$ to represent the overall mode capacities of the fiber segments surrounded respectively by air, by liquid and by the original fiber cladding, from Eq. (1) we get:

$$
N_{g, a r} \approx 7.9 N_{g, c l} \text { and } N_{g, l q} \approx 2.5 N_{g, c l} .
$$

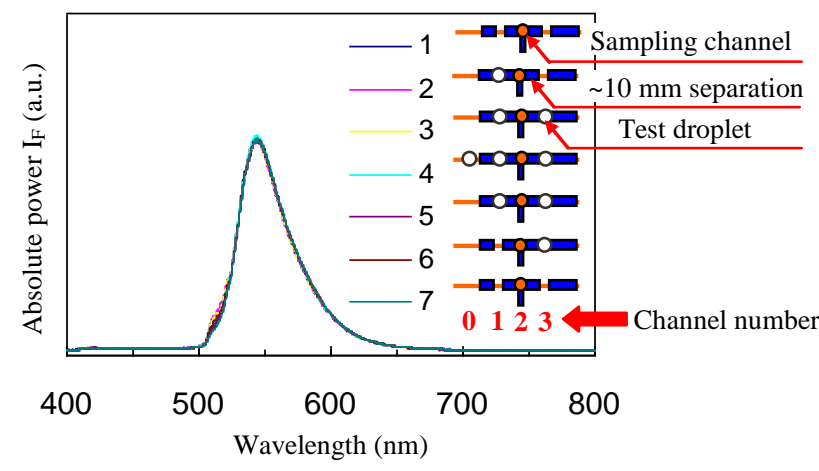

Fig. 2. Experimental verification of zero inter-channel crosstalk.

Equation (2) is illustrated by the histogram in Fig. 3, which has two longitudinal axes, one for the mode capacity and one for the mode order. The validity of the co-existence of these two axes for the same set of bars lies in the fact that wave-optics has assigned each mode a unique number or mode order according to the degree of mode-field confinement in the core. A smaller number indicates a lower-order mode and a better confinement, while a larger number indicates a higher-order mode that is less confined or has greater penetration of its field into the cladding. From this perspective, calculation of the mode capacity of each segment is thus a matter of counting the mode order, starting from the lowest-order mode. Once the highestorder mode is found, the mode capacity is determined. Hence, the heights of the bars could be used to indicate both mode capacity and mode order. This notion will greatly facilitate our discussions below.

Focusing on the guided mode regime associated with the bars in Fig. 3, the short pink bar represents the modes that capture the major portion of EW signal power, by virtue of their greater penetration depth stemming from their higher mode order $[1,5]$. Hence, by defining $N_{g, c l, h}$ as the number of these high-order modes, $N_{g, c l, e f f}$ as the modes that capture EW signal effectively, and $I_{g, c l, h}$ and $I_{g, c l, e f f}$ as their associated power levels, we could write:

$$
N_{g, c l, e f f}=N_{g, c l, h,} \text { and } I_{g, c l, e f f}=I_{g, c l, h} .
$$

On the other hand, the blue bar represents a large number of low-order modes $N_{g, c l, l}$ that are well confined in all three fiber segments, and thus make a negligible contribution $I_{g, c l, l}$ to the overall captured power:

$$
\frac{I_{g, c l, l}}{I_{g, c l, e f f}} \rightarrow 0 \text {, while } N_{g, c l, l}>>N_{g, c l, e f f} \text {. }
$$

The addition of a test droplet (see the inset in Fig. 2) changes the cladding from air to liquid while its removal changes the cladding back to air. As shown in Fig. 3, these events merely switch the mode capacity between the green $\left(N_{g, l q}\right)$ and the orange $\left(N_{g, a r}\right)$ bars. Obviously, the power captured by the modes belonging to the green and the orange bars is inhibited by the original fiber cladding simply because these modes become leaky upon entering the cladded fiber. As a result, the blue $\left(N_{g, c l, l}\right)$ and pink $\left(N_{g, c l, e f f}\right)$ bars, which represent the only mode categories that convey the captured EW power to the spectrometer, remain unaffected in the presence or absence of the test droplets. In other words, achieving zero inter-channel crosstalk requires that the mode capacity of the original clad fiber segment be smaller than that of the air- and liquid-clad segments. While this requirement is implied by Eq. (2), we could rewrite it in a more specific form:

$$
N_{g, c l}<N_{g, a r} \text { and } N_{g, c l}<N_{g, l q} \text {. }
$$

Yet Eq. (5) provides only a partial explanation of zero inter-channel crosstalk operation, based on mode capacity. The aforementioned greater penetration depth of the $N_{g, c l, e f f}$ mode group also has to be taken into account to reveal how $I_{g, c l, e f f}$ is unaffected by the addition or removal of test droplets. Refer again to the histogram in Fig. 3. While all modes of the $N_{g, c l, e f f}$ group are high-order for the clad segment, they become well confined upon entering the liquid- or air-clad segment. For these two segments, the 
modes represented by the pink bar become better confined low-order modes since the green and the orange bars are both far taller than the pink bar, indicating not only a capacity for many more modes but also a far higher mode order for most of their modes. This fact can be further interpreted in terms of ray optics.

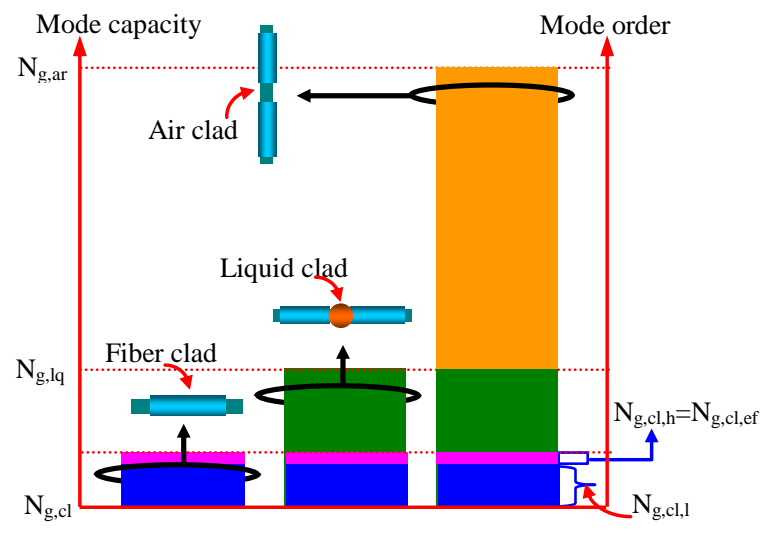

Fig. 3. Histogram showing the mode order and mode capacity of each segment. Note that the bars in blue and pink are permitted in all three segments. Bars in pink, green and orange correspond to the modes with greater ability to capture EW power owing to the larger percentages of their mode fields in the cladding areas.

We define $\Delta_{x}$ as the angle range of the guided rays that is associated with the fiber segment with cladding material $x$ $(x \in(a r, l q, c l))$. For $\Delta_{x}$, we have:

$$
\Delta_{a r}>\Delta_{l q}>\Delta_{c l}
$$

where $\Delta_{x}=90^{\circ}-\theta_{c, x}$ and $\theta_{c, x}$ is the critical angle associated with the cladding material $x$.

From Snell's law and the RIs of the fiber core and cladding given before, with $n_{a r}=1$, we get:

$$
\Delta_{a r}=47^{\circ}, \Delta_{l q}=34^{\circ}, \Delta_{c l}=15^{\circ} .
$$

Equations (6) and (7) are illustrated in Fig. 4, which clearly shows that upon entering the air- or liquid-clad fiber segment, any ray included within $\Delta_{c l}=15^{\circ}$, including any ray near $\theta_{c, c l}$, has its incident angle well below both $\theta_{c, a r}$ and $\theta_{c, l q}$. Since rays near $\theta_{c, c l}$ are responsible for most of the captured EW power, their associated guided modes $N_{g, c l, e f f}$ (pink bar in Fig. 3) are low-order and are well confined in both air- and liquid-clad segments.

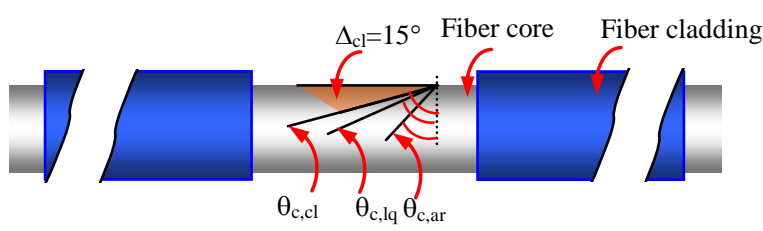

Fig. 4. Critical angles associated with the three cladding materials. Rays included within $\Delta=15^{\circ}$ are guided in all three segments and their associated modes are well confined in both air- and liquid-clad segments. Refer to the text for detail.
Such a confinement can also be described by the penetration depth $\delta_{g, x}$ :

$$
\delta_{g, x}=\frac{\lambda}{4 \pi \sqrt{n_{c o}^{2} \sin ^{2} \theta_{i}-n_{x}^{2}}}, \quad n_{x} \in\left(n_{a r}, n_{l q}, n_{c l}\right),
$$

where $\theta_{i}$ is the incident angle of the guided ray.

Associating $\theta_{i}$ with a mode from the $N_{g, c l, e f f}$ group, Eq. (8) yields:

$$
\delta_{g, a r}<\delta_{g, l q}<\delta_{g, c l} \text {, when } n_{a r}<n_{l q}<n_{c l} .
$$

Hence, upon entering the liquid- or air-clad segment, the EW-field penetration depth of any mode from the $N_{g, c l, e f f}$ group, described in Eq. (8) by the angle $\theta_{i}$, is shallower than would be expected from the normal clad segment. By considering Eqs. (6), (7) and (9) together, we see that the modes corresponding to the angle $\theta_{c, c l}$ and its neighbouring range are so well confined that they are unaffected by the addition/removal of test droplets, resulting in the observation of zero inter-channel crosstalk.

As a discussion, one of the particular advantages offered by the architecture shown in Fig. 1 is its strong ability to capture EW power [3] since all power from the e-fiber in Fig. 1 is used for sample excitation. In contrast, the excitation power of most distributed fiber-optic fluorescence sensors is inherently weak since it is delivered to the samples through the EW fields. Moreover, the platform in Fig. 1 may pinpoint precisely the location of any channel in operation through optical switching and TDM.

In conclusion, we have demonstrated zero inter-channel crosstalk in a 12-channel EW sensing platform. It is expected that more channels could be added without sacrificing the feature of zero inter-channel crosstalk, as long as the condition $n_{l q}<n_{c l}$ is fulfilled. This will be explored in our future work, together with the development of the interrogation system capable of scanning multiple channels.

This work was supported in part by the Natural Sciences and Engineering Research Council of Canada and by the Canada Research Chairs Program.

\section{References}

[1] C.R. Taitt, T.P. Anderson, F.S. Ligler, Biosens. Bioelectron. 20, 2470 (2005).

[2] R. Narayanaswamy, O.S. Wolfbeis, Optical Sensors: Industrial Environmental and Diagnostic Applications (Wolfbeis, Springer, Germany 2004).

[3] J. Ma, W.J. Bock, Opt. Lett. 32, 8 (2007).

[4] H. Chen, J. Ma, J. Chen, W.J. Bock, Opt. Lett. 36, 2092 (2011).

[5] J. Ma, Y. Chiniforooshan, J. Chen, W.J. Bock, W. Hao, Z.Y. Wang, Opt. Lett. 36, 3581 (2011)

[6] D. Gloge, Appl. Opt. 10, 2252 (1971). 